\title{
Review of: "Clinical and molecular factors that impact the efficacy of first-line crizotinib in ROS1-rearranged non-small-cell lung cancer: a large multicenter retrospective study"
}

Tejas Patil ${ }^{1}$

1 University of Colorado Anschutz Medical Campus

Potential competing interests: The author(s) declared that no potential competing interests exist.

This a large retrospective analysis that adds to the pantheon of other ROS1 NSCLC directed studies. While additional data in this space is always welcome, many of the findings are not novel. For example, the finding that crizotinib is better than platinum doublet chemotherapy was the PROFILE-1001 trial. This has since been shown in EUCROSS study as well. While I agree with the reviewers assertion that crizotinib is better than chemotherapy, the authors should perform a separate analysis to see if there is a difference between platinum pemetrexed vs cisplatin/docetaxel, given findings from a separate analysis (Song et al; PMID 27544536) that seemed to suggest that ROS1 NSCLC patients benefit from a pemetrexed regimen. Likewise, the findings regarding brain metastases are not novel (Patil et al JTO 2018; Ou Lung Cancer 2019) and have been reported elsewhere. The data regarding differences between non-CD74 and CD74 fusion partners do conflict with other studies (Xu et al Cancer Med 2020; Shaw et al Annals Oncol 2019), though the authors do mention this discrepancy. 